\title{
EL PROBLEMA DE LA INDUCCIÓN Y LA DEDUCCIÓN
}

\section{Gibson Useche*}

Desde la aparición de los distintos sistemas filosóficos que han elaborado su sistema de pensar el mundo, han tenido su influencia en la construcción o reconstrucción de la ciencia, dejando al interior de las mismas secuelas o vicios, y uno de ellos es precisamente la separación de un tipo de razonamiento de otro. Esta separación la encontramos por ejemplo en las obras de Aristóteles, para quien el único medio seguro de obtención de conocimiento era el Silogismo que lo denominó Demostración General, «el silogismo es una enunciación de la cual, cuando se afirma alguna cosa, se deduce obligatoriamente algo distinto a lo que se afirma y en virtud de lo que existe. Por las palabras: "en virtud de lo que esto existe", entiendo que lo distinto se deduce gracias a ello, y por las palabras "se deduce gracias a ello", que no precisa de ningún otro término para inferirse ineludiblemente». ${ }^{1}$

Así mismo, Aristóteles no dejó de lado la Inducción, como Naturalista Racional y Empírico comprendía la importancia de la experiencia, de la observación en el conocimiento y lo imprescindible del camino de lo singular a lo general.

Entendía que los conceptos generales se forman mediante el estudio de ejemplares singulares, "por lo que se refiere al concepto general de animal, ya no es nada en absoluto, ya se forma después (de la existencia de algunos ejemplares). Lo mismo ocurre cuando se enuncia cualquier otra generalización, tan solo la Inducción, que se denomina ahora completa, puede servimos como forma de demostración rigurosa». ${ }^{2}$

Veamos otro caso donde Aristóteles opone la Inducción a la demostración partiendo de lo general: "Es igualmente evidente que si no hay percepción sensible, tampoco habrá conocimiento, que resulta imposible en este caso adquirir, ya que aprendemos alguna cosa, bien por medio de la Inducción, bien por demostración. La demostración viene de lo general y la inducción de lo particular; sin embargo tampoco lo general puede examinarse sin ayuda de la Inducción. Pero la Inducción es imposible sin la percepción sensible, pues con la experiencia sensible se aprehenden diversas cosas, ya que de otro modo resulta imposible conseguir conocimiento a cerca de ellos. En efecto, lo mismo que el conocimiento adquirido de lo general es imposible sin la Inducción, así mismo el conocimiento adquirido por medio de la Inducción es imposible sin la percepción sensible». ${ }^{3}$

Por tanto, Aristóteles no dio una solución concreta al problema de la Inducción como método que permite conocer lo general a partir de lo singular, pues la doctrina de su época no colocaba en primer plano a la Inducción y además el mundo Antiguo no poseía una ciencia verdadera sobre la naturaleza, su practica no permitía observarla y colocarla a prueba.

Es a finales del siglo XV, cuando aparecen y empiezan a desarrollarse las Ciencias Naturales, surge nuevamente la doctrina de la Inducción. Las Ciencias Naturales surgen

\footnotetext{
* Profesor Universidad Pedagógica Nacional

${ }^{1}$ ARISTÓTELES. Analitica. Edit. Gospolitizdar.

1952. pág. 10.

${ }^{2}$ ARISTOTELES. Del alma. Edit.Golpolitizdar. Moscú, 1973, pág. 4,5

${ }^{3}$ OP-CIT. ARISTÓTELES. Pág. 217,218 
entonces en circunstancias revolucionarias: es la época de la abolición de la Monarquía y la instauración de la República, el nacimiento del capitalismo y con ello la exigencia de una mayor producción de bienes y servicios, el incremento de la técnica, el desarrollo de los conocimientos científico naturales para oponerlos y aplastar la ideología del Feudalismo y la hegemonía de las concepciones religioso-cristianas sobre el mundo que frenaban el desarrollo de la producción.

Hasta finales del siglo XVIII y la primera mitad del siglo XIX, las Ciencias Naturales fueron preferentemente de recopilación dedicadas a describir y registrar los hechos. La ciencia más desarrollada era la Mecánica, la Física empezaba a formarse, la Química y la Biología se hallaban en estado embrionario, «incluso en la primera etapa de su desarrollo, las ciencias naturales se dedicaban a generalizar los hechos de la vida real, a pasar de lo particular a lo general. No podemos decir que en los siglos XVI, XVII y XVIII las ciencias naturales se limitaban solamente a recopilar únicamente los hechos dejando de lado su generalización. Había también generalizaciones, pero se referían principalmente a la sistematización y a la clasificación de los fenómenos de la naturaleza: descripción de los hechos, de los fenómenos y su inclusión en una determinada clase». ${ }^{4}$

Es entonces en la etapa de desarrollo de las Ciencias Naturales Experimentales, donde se fundamentan las principales tesis del método de la Inducción, como la búsqueda de una nueva forma de pensar el mundo que facilitará al hombre conocer los hechos del mundo material y dominar la naturaleza. Sin embargo, la influencia de la Metafísica al interior de la ciencia generó la comprensión errónea de la Inducción y su aislamiento con otras formas de razonamiento, «portadores de ésta tendencia fueron Leonardo Da Vinci, Galileo Galilei, F. Bacon, quienes trataron de aprehender las leyes que presiden el conocimiento de la naturaleza, de poner de manifiesto la dinámica de la trayectoria del saber desde los hechos aislados a las leyes de la naturaleza». ${ }^{5}$

Por ejemplo Leonardo Da Vinci escribía: «Carece de toda veracidad una ciencia que no admita la aplicación de alguna de las ciencias matemáticas o que no esté vinculada a las matemáticas». ${ }^{6}$

Galileo utilizó la Inducción corno parte de su método de investigación, partía del experimento, fórmula generalizaciones, de las cuales deducía nuevos hechos particulares. Las nuevas observaciones comprobaban la deducción hecha y confirmaban la veracidad de las tesis generales formuladas anteriormente.

La Tesis de F. Bacon sobre la Inducción, presenta muchos aspectos correctos sobre el planteamiento general del problema, como el siguiente: «nuestro camino y nuestro método (como hemos dicho claramente y como quiero volverlo a decir), radica en lo siguiente, no inferimos la práctica de la práctica y los experimentos de los experimentos (como los empíricos), sino las causas y los axiomas de la práctica y de los experimentos, y de las causas y los axiomas de la práctica inferimos nuevamente la práctica y los experimentos como fieles interpretaciones de la naturaleza». ${ }^{7}$

Como este otro planteamiento: «la Lógica Escolástica, por ejemplo afirmaba que Caín es mortal, porque el hombre lo es; la Lógica de Bacon por el contrario según cierta expresión de Herzen, trataba de demostrar que el hombre es mortal porque Caín lo era. En la

\footnotetext{
${ }^{4}$ KOPNIN,P.V Lógica Dialéctica. Edit: Grijalbo. México 1966, pág.291

${ }^{5}$ IBID. Pág. 291

${ }^{6}$ DA VINCI, Leonardo. Obras escogidas, Tomo 1. 1935. Págs. 67, 68

${ }^{7}$ BACON F Organum, Sozkguiz. 1938. Pág. 90
} 
Inducción Baconiana el hecho empírico pasa a ser la premisa fundamental y primera del razonamiento» ${ }^{8}$, aspectos positivos que subrayan el papel de la práctica, de la observación, del experimento en las ciencias.

Pero también presenta aspectos incorrectos cuando plantea la Inducción como un método de formación de conceptos seguros, inmodificables, «toda ciencia ha de ser experimental, su esencia se basa en la aplicación del método racional a los datos sensibles. La inducción, el análisis, la comparación, la observación, los experimentos, son las condiciones principales del método racional» ${ }^{9}$.

Al considerar las causas, las formas del fenómeno, los conceptos elaborados, como estancados e invariables, Bacon marca una estrechez en la ciencia con un claro sello metafísico, «la Inducción Baconiana, se basa en el análisis metafísico, unilateral, en la descomposición de la naturaleza en elementos sueltos, aislados. Por ello, Bacon no logró revelar la verdadera trayectoria del conocimiento de lo general, el paso del saber de lo singular a lo general». ${ }^{10}$

Veamos ahora algunos planteamientos de René Descartes, que nos permiten ver la identificación de objetivos con F. Bacon, en cuanto a alcanzar un conocimiento de las cosas y los fenómenos tan útiles en la vida, pero por caminos diferentes.

Se ha mencionado que para Bacon la experiencia constituye el principio del conocimiento y que partiendo de ella, por medio de la Inducción se conocen las causas y las leyes de la naturaleza. Para Descartes, en cambio el principio del conocimiento se basa en la intuición y la deducción, mientras que la experiencia y la Inducción juegan un papel auxiliar «tan solo la Deducción y la intuición son seguras todo lo demás es sospechoso y sujeto a error. Y la intuición es mas segura que la deducción» ${ }^{11}$.

«La deducción sirve para inferir obligatoriamente algo de algo que se conoce anteriormente como fidedigno. La intuición se limita a proporcionar las tesis de partida dela Deducción; la propia Deducción de una tesis partiendo de otra se realiza sobre la base y por medio de la intuición.» ${ }^{12}$.

Avanzando un poco más, dentro de las posiciones de las distintas tendencias que han opuesto la Inducción a la Deducción, pasemos a describir los principales planteamientos del Positivismo Lógico o Empirismo Lógico representado en Schlat, Carnap, Neurath, Frank, Kauffam, Godel y otros.

Por ejemplo Locke afirmaba que «la verdad puede ser descubierta y confirmada tan solo mediante un análisis maduro y adecuado de las propias cosas y no por medio de términos artificiales y procedimientos de argumentación; estos, más que llevar a los hombres al descubrimiento de la verdad, les conduce al empleo sofístico y taimado de palabras ambiguas ${ }^{13}$. En otras palabras, para Locke, los razonamientos más importantes para la ciencia son los que van de lo singular a lo particular, de lo particular a lo general y no los razonamientos que van de lo general a lo particular.

\footnotetext{
${ }^{8}$ OP-CIT. KOPNIN.P.V. pág. 244

${ }^{9}$ IBID. BACON. P

${ }^{10}$ OP CIT P.V. KOPNIN. Pág. 294

${ }^{11}$ IBID P.V.KOPNIN. pág 295

${ }^{12}$ IBID pág. 295

${ }^{13}$ LOCKE 
Por otra parte, B. Russell tratando sobre el mismo tema escribía: «confirmar la Inducción como tal, es imposible, por cuanto se puede demostrar que conduce con la misma frecuencia al error que a la verdad. Sin embargo en casos adecuados conserva suma importancia como medio para elevar la probabilidad de las generalizaciones ${ }^{14}$.

Los positivistas modernos (Lógicos), siguen el mismo camino de los Positivistas, tiñendo de otro color sus principales tesis, para caer en posiciones empiristas, psicologistas o fisiologistas, descartando de plano la Deducción como método para llegar a lo particular partiendo de lo general, «la ley de la inducción, como ya hemos visto, se inicia en la Fisiología y esto nos lleva a enfocar de otro modo los juicios apriorísticos, es decir, de un modo distinto al empleado por Kant. Independiente si existe o no el conocimiento a priori, es indudable que las suposiciones aprioristas existan en cierto sentido. Poseemos reflejos que nuestra mente elabora en suposiciones. El hecho de parpadear nos hace suponer que el contacto de un cuerpo extraño con el ojo, le hace daño. La experiencia nos lleva a semejante suposición, antes de que nos convenza de su veracidad, si esto es así, semejante suposición viene a ser, en cierto sentido, un conocimiento sintético a priori, es decir, se trata del convencimiento, no basado en la experiencia, de la veracidad de un cierto juicio sintético. Nuestra suposición en cuanto a la inducción es, de hecho, análoga a ello. Pero semejantes suposiciones, incluso si son verdaderas, no merecen siquiera el nombre de conocimientos, ya que no siempre son verídicas y por tanto, SIEMPRE PRECISAN SER COMPROBADAS antes de poder ser consideradas como verdaderas ${ }^{15}$ subrayado nuestro.

Podemos decir entonces, que para los positivistas en general, ni la inducción, ni la deducción están relacionadas entre sí, porque según ellos, ambas formas de raciocinio descansan en fundamentos diferentes: la inducción en las propiedades del sistema nervioso de los animales, y la deducción en la necesidad lógica que se infiere de las propiedades de la denominación, ni lo uno ni lo otro están unidos a necesidad que existe en el mundo objetivo.

Hasta aquí, se ha presentado en rasgos generales, las dos tendencias que han existido, y siguen existiendo en la constitución como conocimiento científico, tendencias enfrentadas y recíprocamente excluyentes, mientras se exagera de forma unilateral y exisiva el papel del experimento y la inducción dejando de lado la deducción, en la otra posición, se niega el valor esencial de la experiencia y la inducción basada en ella y se acepta la intuición y la deducción.

Por primera vez, en la historia de la filosofía, el que intenta resolver este problema, de separación y exclusión mutua de la inducción y la deducción, es el filósofo alemán HEGEL, partiendo del materialismo idealista como lo han denominado sus críticos posteriores (Los materialistas dialécticos). «Para HEGEL lo más importante era esbozar las transiciones de una forma de razonamiento a otra, el paso de la deducción a la inducción y el de esta última a través de la analogía, de nuevo a la deducción.»

La propuesta de Hegel para solucionar esta separación consistió en formular una teoría del raciocinio cuya test central plantea que el raciocinio atraviesa tres etapas o grados en su desarrollo: raciocinio de existencia, de reflexión y de necesidad y que no se diferencian por rasgos puramente formales (posición del término medio) sino por su contenido.

\footnotetext{
${ }^{14}$ RUSELL, B. El conocimiento Humano. Moscú, 1957 Pág. 467

${ }^{15}$ KOPNIN, PV. OP-CIT Pág. 299 
Aunque Hegel no separaba la inducción de los restantes tipos de razonamiento, tratando solamente de pensar su lugar en el desarrollo del raciocinio, significa que superó los defectos del silogismo de la totalidad pero no solucionó el carácter problemático de la conclusión inferida por la inducción basada en un experimento incompleto, es decir no superó por completo la subestimación total de la inducción. «El tipo superior de raciocinio era para él la necesidad, deductiva en esencia. Por su valor cognoscitivo coloca la inducción por debajo incluso de la Analogía. Las formas inferiores de la deducción, (el raciocinio de existencia y el de la totalidad) son anteriores a la inducción y la analogía, culminando todo el proceso de desarrollo de los raciocinios.»

Debe tenerse en cuenta que Hegel elaboró la teoría del raciocinio, partiendo del conocimiento existente en su época y que la lógica en ese momento no había descubierto todas las formas de razonamiento empleadas en la práctica del conocimiento científico y que este conocimiento se desarrolla, avanza y por tanto cambian las formas discursivas, se perfeccionan, se desechan formas viejas y surgen otras nuevas.

En conclusión, según sus escritos Hegel no halló un sitio para la práctica en el devenir y las dinámicas de las formas del raciocinio y por ello no pudo determinar el papel desempeñado por la inducción en el conocimiento, pues ninguna forma de raciocinio por si sola puede asegurar la exactitud y la veracidad de las conclusiones.

Continuando en la trayectoria del enfrentamiento y conciliación entre la inducción y la deducción como problemas del conocimiento, veamos como lo plantean los materialistas dialécticos:

En la crítica que hacen a los partidarios del inductismo, señalan que inducción no puede considerarse como un método de razonamiento infalible (en eso coinciden con Karl Popper en su critica al Positivismo lógico), argumentan que las conclusiones obtenidas por inducción son problemáticas por su carácter, luego necesitan ser comprobadas (cosa que no contradice el planteamiento de B. Russell, expuesto anteriormente en el pie de página 14), y que además la práctica de la vida real, el desarrollo científico precisa y modifica las conclusiones obtenidas por vía inductiva. "Si la inducción fuese realmente tan infalible como se dice, ¿Cómo podrían producirse esos desplazamientos radicales de las clasificaciones, tan violentos y frecuentes en el mundo orgánico?. En realidad son el producto mas genuino de la inducción y, a pesar de ello, se anulan los unos a los otros».

Toman el ejemplo de Lavoiser, que se equívoca al deducir que el oxigeno estaba presente en todos los ácidos partiendo de la inducción y como posteriormente con el hallazgo de que él ácido clorhídrico no contiene oxígeno se refuta esa conclusión, y se establece que es el hidrógeno el que está presente en todos los ácidos. Así mismo señala que la ley de Boyle-Mariotte ha sido refutada por la física moderna al establecer que los gases a temperaturas críticas y con una presión del orden de centenares de atmósferas adquieren un mayor volumen del que les corresponde, al aplicar dicha ley.

Refiriéndose al problema del empleo de la inducción no como totalidad en las matemáticas, describen como ella es imposible sin razonamientos inductivos, y que como teoría no están acabadas. Concluyen que en las matemáticas como en otras ciencias la inducción esta vinculada a la deducción, que no existe ninguna esfera del conocimiento científico que no procure entender la tesis general a diversos objetos, fenómenos o casos concretos, en otras palabras procurar la coincidencia de los hechos y, los fenómenos concretos con las leyes establecidas, donde la deducción desempeña un papel 
fundamental en la comprobación de los enunciados de las teorías o hipótesis obtenidas por medio de la analogía y la inducción.

Para los materialistas dialécticos, la inducción y la deducción interactúan constantemente en el proceso del conocimiento, no están abruptamente separados en polos opuestos como lo han colocado las demás tendencias desde su posición filosófica.

"Inducción y deducción forman necesariamente un todo, ni más ni menos que la síntesis y el análisis. En vez de exaltar unilateralmente la una a costa de la otra, hay que procurar poner a cada una en el lugar que le corresponde, lo que solo puede hacerse sino se pierde de vista que ambas forman una unidad y se complementan naturalmente".

Dos ejemplos muy claros, que muestran, en forma práctica, como se manifiesta esa unidad, se encuentran en el análisis de la mercancía hecha por Carlos Marx y en la esencia del Imperialismo como fase superior del capitalismo elaborada por Vladimir Ilich Lenin.

Desde esta óptica, superan los planteamientos hechos por Hegel en su teoría del raciocinio y formulan la unidad dialéctica de dos aspectos de un mismo proceso del pensamiento en forma de raciocinio, donde su unidad y conversión reciproca no se excluyen sino que suponen su oposición como un hecho de la realidad y no del formalismo lógico. Para ellos la inducción es un raciocinio que pasa del conocimiento de un grado de generalización menor a otro conocimiento de mayor generalización y la deducción es un raciocinio que pasa del conocimiento de un grado mayor de generalización a otro conocimiento de menor generalización, es decir que sino los considera como raciocinios opuestos no tendría objeto que la una complementara a la otra, en el proceso de obtener la verdad.

Otro aspecto que sobresale en el tratamiento de este problema, es que trascienden de la mera formulación teórica y práctica de la unidad y oposición de la inducción y la deducción, para indicar las existencias de otras formas de razonamiento que se relacionan con ellas pero que son distintas.

«Y es que gente se ha acostumbrado de tal modo a contraponer la inducción y la deducción, que reduce todas las formas lógicas de discurrir a estas dos, sin darse cuenta:

- de que, inconscientemente aplica bajo esos nombres otras formas discursivas,

- $\quad$ de que renuncia a toda riqueza de las formas de discurrir, cuando no pueden encuadrarlas a la fuerza en cualquiera de aquellas dos, y,

- de que, con ello convierte en una pura necedad las dos formas de la inducción y la deducción»

Para argumentar la importancia y el valor cognoscitivo del raciocinio en las diversas etapas del desarrollo del conocimiento científico las clasifica teniendo en cuenta tres rasgos: la composición, la veracidad de la conclusión y la tendencia del proceso deductivo.

Por su composición establecen que todos los racionamientos pueden dividirse en simples, si están formados por un solo razonamiento (sea deductivo o inductivo) y en complejos, si están formados por razonamientos simples de una o de diversas formas. 
Por el grado de la veracidad de la conclusión, indican que todos los razonamientos pueden dividirse en: racionamientos de probabilidad donde la conclusión es un juicio verídico.

Aquí señalan la importancia de las formas discursivas verídicas, para las demostraciones estrictas y las formas que no proporcionan conclusiones fidedignas para la formulación de supuestos científicos que permitan establecer nuevas hipótesis para la explicación de hechos ya conocidos o por conocer.

Según la tendencia del proceso deductivo (1) dividen los razonamientos en: traductivos o sea aquellos que pasan de conocimiento de un grado de generalización al conocimiento del mismo grado; inductivos los que pasan del conocimiento de un grado de generalización menor a otro mayor; deductivos los que pasan del conocimiento de un grado de generalización mayor a otro de menor grado. 